\title{
Wirebonding SVX Prime Silicon Ladders using the K\&S 1478 Wirebonder
}

\author{
Albert Dyer \\ Fermi National Accelerator Laboratory \\ P.O. Box 500, Batavia, Illinois 60510
}

September 1993 


\section{Disclaimer}

This report was prepared as an account of work sponsored by an agency of the United States Government. Neither the United States Government nor any agency thereof, nor any of their employees, makes any warranty, express or implied, or assumes any legal liability or responsibility for the accuracy, completeness, or usefulness of any information, apparatus, product, or process disclosed, or represents that its use would not infringe privately owned rights. Reference herein to any specific commercial product, process, or service by trade name, trademark, manufacturer, or otherwise, does not necessarily constitute or imply its endorsement, recommendation, or favoring by the United States Government or any agency thereof. The views and opinions of authors expressed herein do not necessarily state or reflect those of the United States Government or any agency thereof. 
WIREBONDING SVX PRIME SILICON LADDERS

USING THE K\&S 1478 WIREBONDER

ALBERT DYER

X3863

MARCH 1, 1993

TM1858 
PAGE

1.

2.

2.

2.

3.

3.

3.

5.

6.

8.

9.

9.

11.

11.

11.

14.

17.

19.

22.

24.

27.

29.

32.

35.

37.

39.

42.

44.

47.

47.

50.

52.

55.

58.

58.

60.

63.

66.

69.
TABLE OF CONTENTS

SECTION

\section{POWER UP}

TOOL CAL MODE

SET THETA ZERO

CALIBRATE X-Y TABLE

TOOL CALIBRATION

ADJUST FOCUS

ADJUST LIGHTING

THAEADING THE WIEE THPROUGH THE TOOL

MANUAL MODE

TEACH THE WORKHOLDER THE LOAD POSTIION

USING THE LOAD POSITION MANUALLY

TEACH MODE

REFERENCE SYSTEMS

XYOPOINT

REFERENCE SYSTEM 1

REFERENCE SYSTEM2

REFERENCE SYSTEM 3

REFERENCE SYSTEM 4

REFERENCE SYSTEM 5

REFERENCE SYSTEM 6

REFERENCE SYSTEM7

REFERENCE SYSTEM 8

REFERENCE SYSTEM 9

REFERENCE SYSTEM 10

REFERENCE SYSTEM 11

REFERENCE SYSTEM 12

REFERENCE SYSTEM 13

REFERENCE SYSTEM 14

INNER ROWS

REFERENCE SYSTEMS 1 \& 3

REFERENCE SYSTEMS 5 \& 7

REFERENCE SYSTEMS $9 \& 11$

REFERENCE SYSTEMS $9 \& 13$

OUTER ROWS

REFERENCE SYSTEMS 2 \& 4

REFERENCE SYSTEMS $6 \& 8$

REFERENCE SYSTEMS $10 \& 12$

REFERENCE SYSTEMS $10 \& 14$

FLOPPY MODE 


\section{K\&S 1478 REFERENCE GUIDE}

\section{POWER VP}

1. Rotate counter-clockwise the dry nitrogen valve.

2. Install a test device onto the workholder.

3. Install, then align the workholder onto the X-Y table.

4. Lift the vacuum pump switch to $O N$.

5. Lift Main Breaker to ON.
A minimum of 60 psi is required for stable operation.

Used for calibration and setting bonding parameters.
EMERGENCY STOP bUtton shines. ERROR button shines (this does not mean there is an error).

6. Swing open the steel front panel that shields the front of the computer.

7. Lift the +5 VDC switch to ON.

Display shows : NO POWER

The CAL LED and P.R.S. LED are lit on the keyboard.

8. Press the reset switch (SW1) on the edge of the bonder MPU board.

Display shows: TESTING

(approx. 25-30 sec.), SELFTEST (tests RAM \& PROMS, all MODE LED's are pulsed active) and then: KILRAM?

9. Close the front panel.

10. If KILRAM is YES, press the ACCEPT key.

11. Display shows: NO POWER

12. The CAL LED and P.R.S. LED are lit on the keyboard.

13. If $K I L R A M$ is NO, press the ENTER button.

14. Display shows: NO POWER
All program information is deleted.

\section{Release the EMERGENCY} STOP button.

Display shows: + THETA-O

Current tool calibration and any programs taught are retained.

Release the EMERGENCY STOP button. 
15. The CAL LED and P.R.S. LED are lit on the keyboard.

16. If the bonding wire has to be threaded through the tool,
Display shows: +THETA-O skip to THREADING THE WIRE THROUGH THE TOOL.

\section{IOOL CAL MODE}

1. Press the CAL key on keyboard.

A lit LED next to the CAL key denotes the active mode.

2. If accurate tool calibration information is already in memory:

press the ACCEPT key to skip the tool calibration procedure.

3. If step 2 is not appropriate, continue the TOOL CALIBRATION procedure.

\section{SET THETA ZERO}

1. Display shows : +THETA-O

The computer is ready to receive the theta zero position of the bond head.

2. If accurate theta zero information is already in memory,

press the ACCEPT key.

3. If ACCEPT is not appropriate, see:

MODEL 1478 VOLUME 1

OPERATION MANUAL 8.1.2 SET THETA ZERO.

\section{CALIBRATE X-Y TABLE}

1. Display shows: $+T B L S I Z$

The computer is ready to receive information on the travel limits of the $X-Y$ table.

2. If X.Y limits are aiready in memory, press the ACCEPT key.

3. If ACCEPT is not appropriate, press the ENTER button.

The X-Y table travels to its four limits. The display shows SEEKEDGE during this period. It then travels to the table's $X-Y$ center.

4. The display shows: + ${ }^{*}$ NTER* 


\section{ADJUST FOCUS}

1. The focus can be adjusted anytime.

\section{ADJUST LIGHTING}

1 If the SPECIAL FUNCTION key is pressed prior

2. Press the SPECIAL FUNCTION key,

3. Press the ACCEPT key when the lighting is suitable.

4. If tool calibration is necessary,

5. Looking at the monitor,

6. Press the ENTER button.

7. Displays shows:

8. Looking at the monitor,

9. Press the ENTER button.
By pressing the number keys $(0-9)$ when the " + " is on the display until the desired image sharpness is achieved. Halfstep focus can be achieved by pressing the left upper most key on the keypad (un-marked) as necessary.

to the 0 (PRS LGH) key, the lighting on the device can be adjusted. This enhances the contrast for the best image viewing on the monitor.

the 0 (PRS LGH) key and then the CHANGE key, to change the lighting pattern.

the workholder with a throwaway device must be installed onto the $X-Y$ table.

use the encoders to move the $X-Y$ table to a blank area large enough to perform a tool calibration (the area within the four corners).

The tool produces a 2nd bond footprint in the following order: 9, 6, 3 and 12 o'clock positions.

+ CAL PTY

use the encoders to place the crosshair precisely over the center of the 9 o'clock position (the first calibration point).

Display shows: + CAL PT2 
10. Looking at the monitor,

11. Press the ENTER button.

12. Looking at the monitor,

13. Press the ENTER button.

14. Looking at the monitor,

15. Press the ENTER button.

16. Display shows: +EYE PT

17. Adjust the lighting if necessary.

18. Press the ACCEPT key when the lighting is suitable.

19. Press the ENTER button. use the encoders to place the crosshair precisely over the center of the 6 o'clock position (the second calibration point).

Display shows: + CAL PT3

use the encoders to place the crosshair precisely over the center of the 3 o'clock position (the third calibration point).

Display shows: + CAL PT4

use the encoders to place the crosshair precisely over the center of the 12 o'clock position (the fourth calibration point).

Press the CHANGE key until the second smallest "BOX" is shown on the monitor. Using the encoders, move the $X-Y$ table until a area with contrast changes and lines in the XY axis are found within the selected "BOX". This will yield a good eyepoint.

Press the SPECIAL FUNCTION key, the o (PRS LGH) key and then the CHANGE key, to change the lighting pattern.

Display shows: LEARNING and then SCALING if the P R S accepted the eyepoint. This calibrates video pixels to $X-Y$ table pulses $(9$ pulses $=1 \mathrm{mil}$ in $Z$ mode. 12.7 pulses $=1 \mathrm{mil}$ in $X$ or $Y$ mode). 
20. The display alternately shows:

+EYE PT, the program identifier and CAL DONE if all TOOL CALIBRATION bonds were within an acceptable distance and if the angular tolerances were acceptable.

21. After a successful calibration, press the MANUAL mode key or,

22. Press the EMERGENCY STOP button.

The button will shine, the $Z$ rod solenoid can be heard latching and the servo motors have no power.

\section{IHREADING THE WIRE THROUGH THE TOOL}

1. Press the MANUAL mode key.

Confirm the MANUAL mode LED is lit.

2. Using the encoders, move the workholder from under the bonding tool.

CAUTION: do not leave the $X-Y$ table at any $X-Y$ stop limit. This can cause the machine to lockup. Ihis is a fatal error.

3. Press the EMERGENCY STOP button.

4. Manually turn the bonding head,

5. Press the OPEN CLAMP key on the keyboard one time.
The button will shine, the $Z$ rod solenoid can be heard latching and the servo motors have no power.

to about the 8 o'clock position (or any convenient angle) from which to pull the wire from the bottom of the transolucer through the heel/toe of the tool.

The OPEN CLAMP LED shines denoting the wire clamp is open. Place the bonding wire between the wire guides, then between the jaws of the clamp. The wire clamp will remain open for approximately 30 seconds and then automatically close to prevent the clamp solenoid from overheating. If the lit LED next to the OPEN CLAMP key goes off, the clamp has closed. 
6. Leave a small length of wire,

\section{MANUAL MODE}

Note: Bonding parameters can be changed while in MANUAL mode.

1. Press the MANUAL mode key.

2. Release the EMERGENCY STOP button.

3. Adjust the focus.

4. Adjust the lighting.

7. To display the loop height of the wires,

8. The current setting for the inner row is \#\#\#. extending from the toe of the bonding tool. This will identify CAL PT 1 when preforming a TOOL CALIBPATION.
However, the changes will have no effect on programs used during AUTO mode. If a parameter is changed while the computer is in TEACH mode or SEMI-AUTO mode, the changed parameter will be in effect during AUTO mode.

Confirm the MANUAL mode LED is lit.

Display shows: +BND PT 1

By pressing the number keys $(0-9)$ when the " + " is on the display until the desired image sharpness is achieved. Halfstep focus can be achieved by pressing the left upper most key on the keypad (un-marked) as necessary.

Press the SPECIAL FUNCTION key, the 0 (PRS LGH) key and then the CHANGE key, to change the lighting pattern.

use the encoders to move the $X-Y$ table so that bond point 1 is under the crosshair.

Press the LOOP HEIGHT key. LOOP HEIGHT is different between the inner row and outer row. Consult the LOG BOOK for current parameters.

Press \# \# \# keys. 
9. Display shows: \#\#

10. Display shows: ! $A 1 D \quad 1 W$

11. Press the ACCEPT key.

12. Display the contact velocity of the bonding head.

13. Press the 6 key.

14. Press the ACCEPT key.

15. Displays shows: ! A $101 \mathrm{~W}$

16. Display the loop profile.

17. Display shows: \% CLM TIP

18. Display shows: \% TER

19. Display shows: $+B N D P T 1$

20. Press the ENTER button.

21. Looking at the monitor,

22. Display the contact velocity of the bonding head.

23. Press the 6 key.
Press the ACCEPT key.

Press the ONE DEVICE key.

Press the SPECIAL FUNCTION key and then the LOOP HEIGHT (CVL) key.

Display shows: 4 .

This is the contact velocity of the bonding head. The current setting for CVL is 6 .

Display shows: 6

Press the ONE DEVICE key.

Press the SPECIAL FUNCTION key and then the TIME (CLM) key. The loop profile can be changed by changing the point during the bonding cycle where the wire is clamped. Use the TER position.

Press the CHANGE key until TER is displayed.

Press the ACCEPT key.

Display shows: $+B N D$ PT 2

use the encoders to move the $X-Y$ table so the crosshair displayed on the monitor is over the center of the second bond point of wire 1.

Press the SPECIAL FUNCTION key and then the LOOP HEIGHT (CVL) kөy.

Display shows: 4 .

This is the contact velocity of the bonding head. The current setting for CVL is 6 .

Display shows: 6 
24. Press the ACCEPT key.

25. Press the ENTER button.

26. The crosshair on the monitor,

27. Press the MWD (AUT/MAN) key.

28. Display shows: $\% O N$
Display shows: + BND PT 2

The bonder will automatically bond one wire between the two points.

will return to the first bond to enable the operator to inspect the first bond.

The Missing Wire Detector system is automatically enabled during power-up. To confirm that MWD is enabled, press MWD (AUT/MAN) key.

Press the ACCEPT key. MWD "learns" the bond parameters of the first 64 wires placed for evaluation of bond strength. MWD will also stop the bonding process in the AUTO mode should a wire not fit into the "learned" bonding parameters or not bond onto the pad at all.

\section{IEACH THE WORKHOLDER THE LOAD POSITION}

1. Release the EMERGENCY STOP button.

2. Confirm the MANUAL mode LED is lit.

3. Confirm the P.R.S. mode LED is lit.

4. Looking at the monitor, use the encoders to place the crosshair precisely over the center of the first bond point.

5. Press the SPECIAL FUNCTION key and then press the MWD (AUT/MAN) key.

6. Look at the display.

If the display shows \% MAN $W H$, press the ACCEPT key.

7. If not, press the CHANGE key

until \% MAN WH is in the display, then press the ACCEPT key.

8. Press the SPECIAL FUNCTION key and then press the W/H TEACH key. 
9. Display shows:

10. Looking at the monitor,

11. Press the ENTER button.

12. Display shows: +BND PT 1

\section{USING THE LOAD POSITION MANUALLY}

1. To enable the load position feature, press:

2. Press the ACCEPT key.

3. Press the INDEX button.

4. Press the INDEX button.

5. Select a operating mode.

6. Latch down the EMERGENCY STOP button.
TEACH WH for one second, then + UNLDPS

use the encoders to move the $X-Y$ table to any desired position. NOTE: movement must be less than 10" of table travel.

Display flashes POS TAUT for one second. The table moves to bond point 1 .

the SPECIAL FUNCTION key and then press the RECORD (IDX) key. If the display does not show \% IDX ON, press the CHANGE key until it does.

The workholder moves to the load position and stops.

The workholder returns to the prior position and stops.

To enter into a operating mode press the key next to the mode desired. The LED is lit to confirm the active mode selected.

The button will shine and the $Z$ rod solenoid can be heard latching. Use this feature anytime to safely halt all operations.

\section{IEACH MODE}

1. Release the EMERGENCY STOP button.

2. Press the TEACH mode key.

The LED is lit to confirm the active mode. 
3. Confirm the P.R.S. mode is active.

4. Display shows: ICLR PRG

5. To save existing programs and bonding parameters see: ELOPPY MODE.

6. Display shows:

7. Adjust the lighting.

8. Press the ACCEPT key when the lighting is suitable.

9. Adjust the focus.

10. If the SPECIAL FUNCTION key is pressed

11. Press the ACCEPT key.
Pattern Recognition System is active if LED is lit.

Press the CLEAR key.

All previous program

information is deleted.

HYBRID. If the display shows $\%$ IC, press the CHANGE key then press the ACCEPT key so that $\% H Y B R I D$ is displayed.

Press the SPECIAL FUNCTION key, the 0 (PRS LGH) key and then the CHANGE key, to change the lighting pattern.

By pressing the number keys $(0-9)$ when the " + " is on the display until the desired image sharpness is achieved. Halfstep focus can be achieved by pressing the left upper most key on the keypad (un-marked) as necessary.

before Z ZERO (Focus) key is pressed, in any mode except CAL mode the automatic focus feature can be programmed. If the display shows $+X Y O$ before SPECIAL FUNCTION and $Z$ ZERO (FOCUS) is pressed, all previously programmed focus levels will be changed. 


\section{REEERENCE SYSTEMS}

\section{XYO POINT}

1. Display shows: $+X Y O$

Looking at the monitor use the encoders to move the $X-Y$ table so the crosshair on the monitor is positioned under the desired XYO point. Use the ground trace on the left side of the 1st wafer. This is also the $1 \mathrm{st}$ operator reference point of reference system 1.

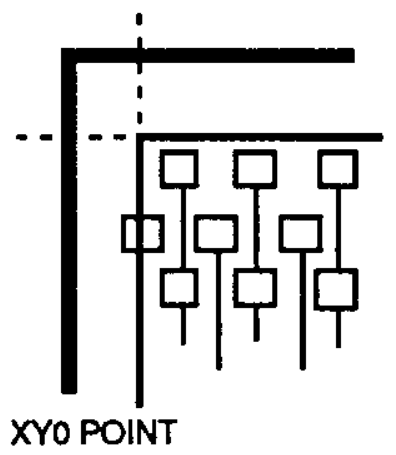

2. Press the ENTER button.

Display shows: ? RSYS there are fourteen reference systems on a layer 1 device.

3. Press the 14 keys.

4. Press the ACCEPT key.

\section{REFERENCE SYSTEM 1}

1. Display shows: ?\#PT 1

2. Press the number 2 key.

The computer is asking for the number of operator reference points in that will be taught for the first reference system. Use 2 reference points.

Display shows: $\quad ? 2=2$ 
3. Press the ACCEPT key.

4. Press the ENTER button.

5. Enter the 2nd operator reference point.

6. Press the ENTER button.

7. Press the ACCEPT key.

8. Display shows: ? EYE 1
Display shows: +REF PTI

Enter the 1st operator

reference point. Use the same reference point that was used for XYO for REF PTI.

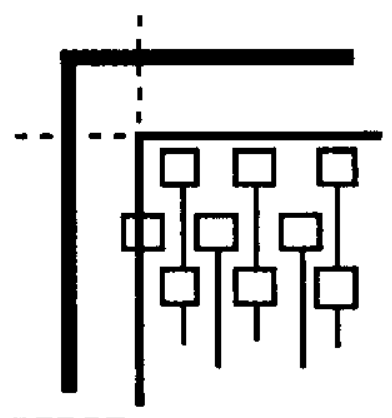

REF PT 1

Display shows: $+R E F \quad P T 2$ Looking at the monitor, use the encoders to move the $X-Y$ table so the crosshair displayed on the monitor is at the right corner of the wafer.

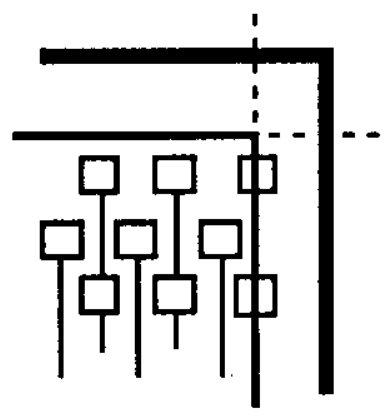

REF PT 2

Display shows: \% DIE TOL The computer is asking for tolerance parameters for the reference system. This has already been defined.

The computer is asking to define how many eye points in the reference system. Use 2 eye points. Pattern Recognition System (P.R.S.). 
9. Press the number 2 key.

10. Press the ACCEPT key.

Display shows: +EYE PTf

11. Press the CHANGE key.

This will reduce the BOX. Use the 2nd smallest BOX.

12. Adjust the focus.

By pressing the number keys $(0-9)$ when the " + " is on the display until the desired image sharpness is achieved. Halfstep focus can be achieved by pressing the left upper most key on the keypad (un-marked) as necessary.

13. Looking at the monitor,

use the encoders to move the $X-Y$ table so the BOX displayed on the monitor is inside the left corner of the wafer.

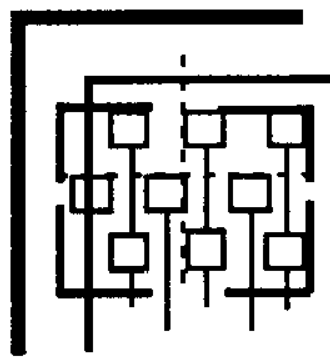

EYE PT 1

14. To evaluate the potential eyepoint:

press the ERROR button.

15. Display shows: SCANNING

When scanning is complete, the display flashes $X=$ (plus a number) and then $Y=$ (plus a number). The difference between $X-Y$ is the quality number. The best eyepoints have two high numbers which are nearly identical.

16. The display automatically returns to $+E Y E P T 1$

17. Press the ENTER button.

Display shows: LEARNING while the $X-Y$ table scans the area in the BOX. 

encoders to move the X-Y table so the BOX displayed on the monitor is just inside the right corner of the wafer.

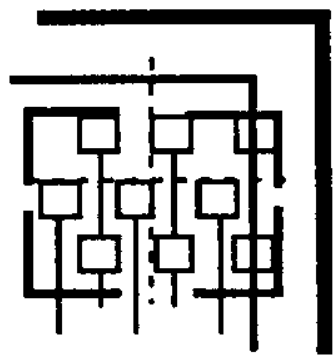

EYE PT 2

19. Press the CHANGE key.

20. Press the ENTER button.

\section{REFERENCE SYSTEM 2}

1. Display shows: ?\#PT 2

2. Press the number 2 key.

3. Press the ACCEPT key.
This will reduce the BOX. Use the 2nd smallest BOX.

Display shows: $L E A R N I N G$ while the $X-Y$ table scans the area in the BOX.

The computer is asking for the number of operator reference points in that will be taught for the second reference system. Use 2 reference points.

Display shows: ? $2=2$

Display shows: +REF PTI The computer is asking for the first operator reference point. 
4. Looking at the monitor,

5. Press the ENTER button.

6. Looking at the monitor,

7. Enter the 2nd operator reference point.

8. Press the ENTER button.

9. Press the ACCEPT key. use the encoders to move the $X-Y$ table so the crosshair displayed on the monitor is at the left corner of the trace.

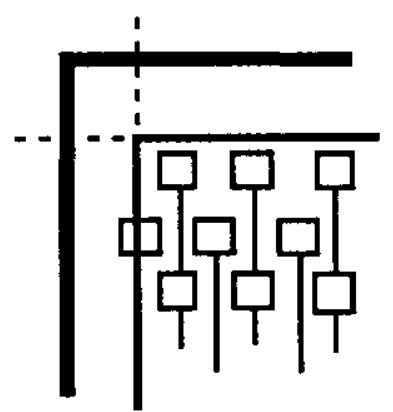

REF PT 1

Display shows: $+R E F \quad P T 2$ The computer is asking for the second operator reference point.

use the encoders to move the $X-Y$ table so the crosshair displayed on the monitor is at the right corner of the trace.

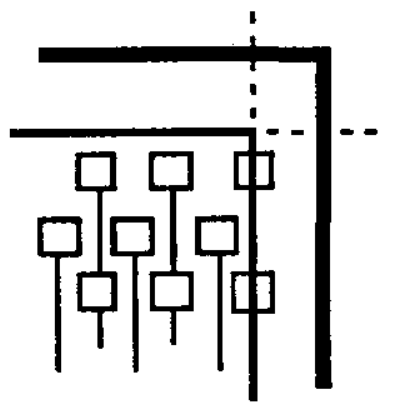

REF PT 2

Display shows: \% DIE TOL The computer is asking for tolerance parameters for the reference system. This has already been defined. 
10. Display shows: ? EYE 2

11. Press the number 2 key.

12. Press the ACCEPT key.

13. Press the CHANGE key.

14. Adjust the focus.

15. Looking at the monitor,

16. Press the ENTER button.
The computer is asking to define how many eye points in the reference system. Use 2 eye points.

Display shows: +EYE PT1

This will reduce the BOX. Use the 2nd smallest BOX.

By pressing the number keys $(0-9)$ when the " + " is on the display until the desired image sharpness is achieved. Halfstep focus can be achieved by pressing the left upper most key on the keypad (un-marked) as necessary.

use the encoders to move the $X-Y$ table so the BOX displayed on the monitor is just inside the left corner of the wafer.

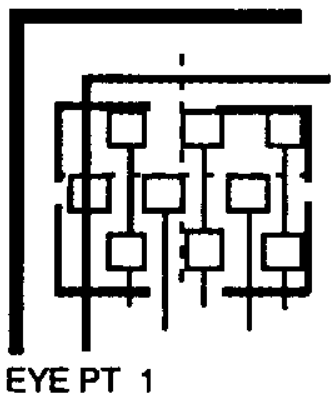

Display shows: LEARNING while the $X-Y$ table scans the area in the BOX. 
17. Display shows: $+E Y E \quad P T 2$

18. Press the ENTER button.

\section{BEFERENCE SYSTEM 3}

1. Display shows: ?\#PT 3

2. Press the number 2 key.

3. Press the ACCEPT key.

4. Looking at the monitor,
Looking at the monitor, use the encoders to move the $X-Y$ table so the BOX displayed on the monitor is just inside the right corner of the wafer.

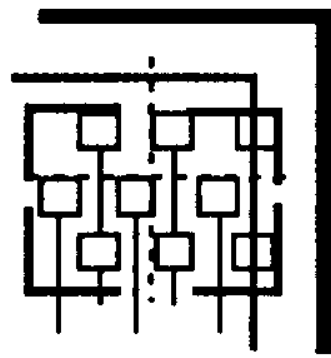

EYE PT 2

Display shows: LEARNING while the $X-Y$ table scans the area in the BOX.

The computer is asking for the number of operator reference points in that will be taught for the third reference system. Use 2 reference points.

Display shows: ? $2 \times 2$

Display shows: +REF PT1 The computer is asking for the first operator reference point.

use the encoders to move the $X-Y$ table so the crosshair displayed on the monitor is at the left corner of the trace.

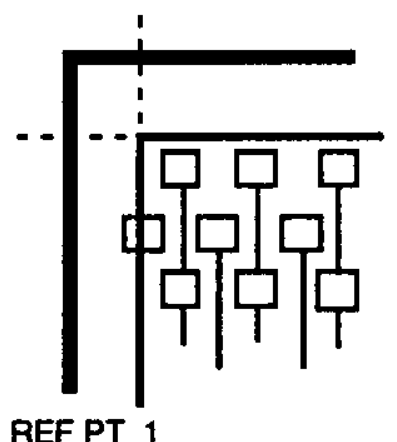

REF PT 1 
5. Press the ENTER button.

6. Looking at the monitor,

7. Enter the 2nd operator reference point.

8. Press the ENTER button.

9. Press the ACCEPT key.

10. Display shows: ? EYE 2

11. Press the number 2 key.

12. Press the ACCEPT key.

13. Press the CHANGE key.

14. Adjust the focus.
Display shows: +REF PT2 The computer is asking for the second operator reference point.

use the encoders to move the $X-Y$ table so the crosshair displayed on the monitor is at the right corner of the trace.

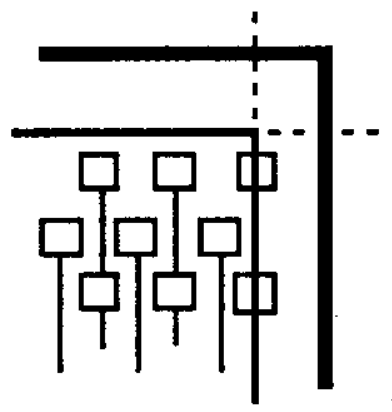

REF PT 2

Display shows: \% DIE TOL The computer is asking for tolerance parameters for the third reference system. This has already been defined.

The computer is asking to define how many eye points in the reference system. Use 2 eye points.

Display shows: +EYE PT1

This will reduce the BOX. Use the 2nd smallest BOX.

By pressing the number keys $(0-9)$ when the " + " is on the display until the desired image sharpness is achieved. Halfstep focus can be achieved by pressing the left upper most key on the keypad (un-marked) as necessary. 
15. Looking at the monitor,

16. Press the ENTER button.

17. Display shows: +EYE PT2

18. Press the ENTER button.

\section{REFERENCE SYSTEM_4}

1. Display shows: ?\#PT 4

2. Press the number 2 key.

3. Press the ACCEPT key. use the encoders to move the $X-Y$ table so the BOX displayed on the monitor is just inside the left corner of the wafer.

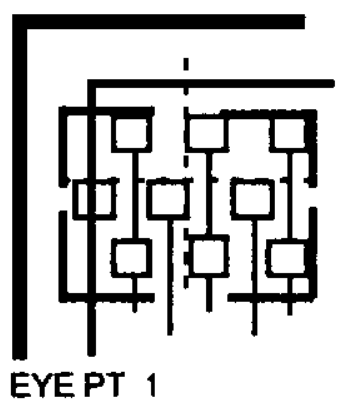

Display shows: LEARNING while the $X-Y$ table scans the area in the BOX.

Looking at the monitor, use the encoders to move the $X-Y$ table so the BOX displayed on the monitor is just inside the right corner of the wafer.

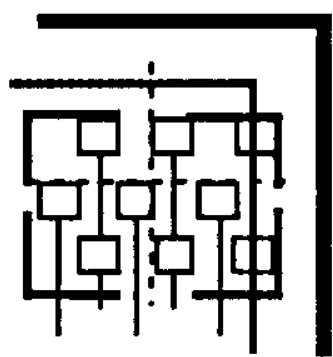

EYE PT 2

Display shows: LEARNING while the $X-Y$ table scans the area in the BOX.

The computer is asking for the number of operator reference points in that will be taught for the fourth reference system.

Use 2 reference points.

Display shows: $\quad 2=2$

Display shows: +REF PT1 The computer is asking for the first operator reference point. 
4. Looking at the monitor,

5. Press the ENTER button.

6. Looking at the monitor,

7. Enter the 2nd operator reference point.

8. Press the ENTER button.

9. Press the ACCEPT key.

10. Display shows: ? EYE 2 use the encoders to move the $X-Y$ table so the crosshair displayed on the monitor is at the left corner of the trace.

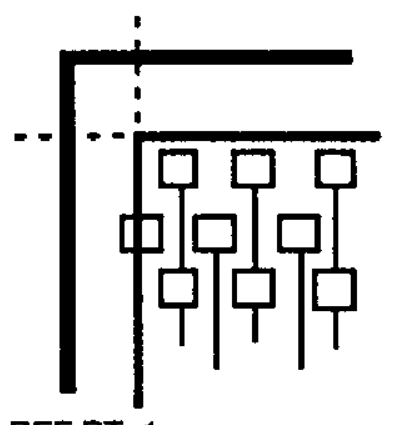

REF PT 1

Display shows: +REF PT2 The computer is asking for the second operator reference point.

use the encoders to move the $X-Y$ table so the crosshair displayed on the monitor is at the right corner of the trace.

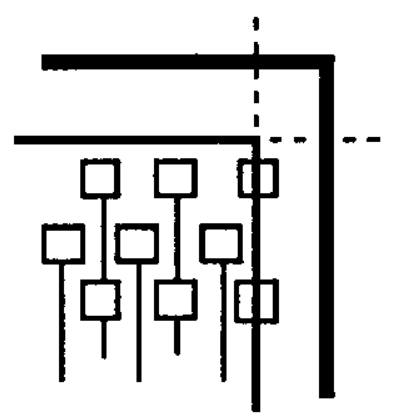

REF PT 2

Display shows: \% DIE TOL The computer is asking for tolerance parameters for the fourth reference system. This has already been defined.

The computer is asking to define how many eye points in the reference system. Use 2 eye points. 
11. Press the number 2 key.

12. Press the ACCEPT key.

13. Press the CHANGE key.

14. Adjust the focus.

15. Looking at the monitor,

16. Press the ENTER button.

17. Display shows: $+E Y E \quad P T 2$
Display shows: $+E Y E \quad P T 1$

This will reduce the BOX. Use the 2nd smallest BOX.

By pressing the number keys $(0-9)$ when the " + " is on the display until the desired image sharpness is achieved. Halfstep focus can be achieved by pressing the left upper most key on the keypad (un-marked) as necessary.

use the encoders to move the $X-Y$ table so the BOX displayed on the monitor is just inside the left corner of the wafer.

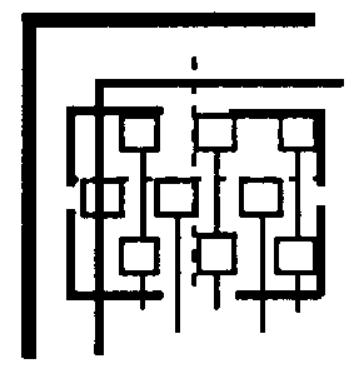

EYE PT 1

Display shows: LEARNING while the $X-Y$ table scans the area in the $B O X$.

Looking at the monitor, use the encoders to move the $X-Y$ table so the BOX displayed on the monitor is just inside the right corner of the wafer.

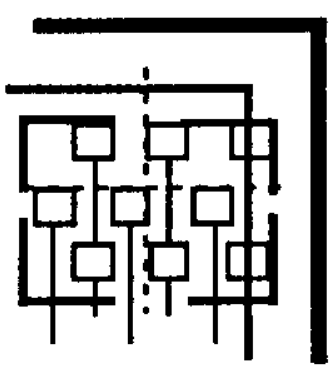

EYE PT 2 
18. Press the ENTER button.

\section{BEFERENCE SYSTEM 5}

1. Display shows: ?\#PT 5

2. Press the number 2 key.

3. Press the ACCEPT key.

4. Looking at the monitor,

5. Press the ENTER button.
Display shows: LEARNING while the $X-Y$ table scans the area in the BOX.

The computer is asking for the number of operator reference points in that will be taught for the fifth reference system.

Use 2 reference points.

Display shows: $\quad ? \quad 2=2$

Display shows: +REF PT1 The computer is asking for the first operator reference point.

use the encoders to move the $X-Y$ table so the crosshair displayed on the monitor is at the left corner of the trace.

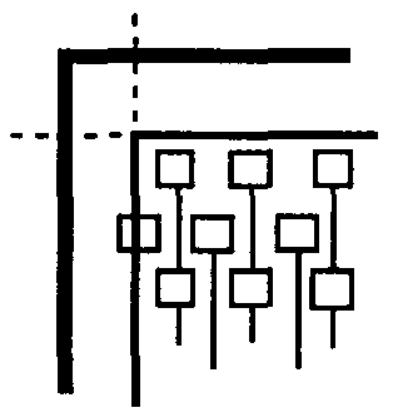

REF PT 1

Display shows: $+R E F \quad P T 2$ The computer is asking for the second operator reference point. 
6. Looking at the monitor,

7. Enter the 2nd operator reference point.

8. Press the ENTER button.

9. Press the ACCEPT key.

10. Display shows: ? EYE 2

11. Press the number 2 key.

12. Press the ACCEPT key.

13. Press the CHANGe key.

14. Adjust the focus. use the encoders to move the $X-Y$ table so the crosshair displayed on the monitor is at the right corner of the trace.

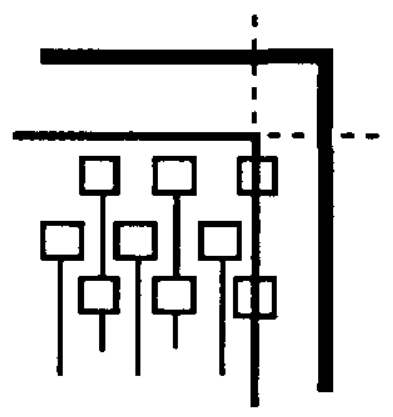

REF PT 2

Display shows: \% DIE TOL The computer is asking for tolerance parameters for the fifth reference system. This has already been defined.

The computer is asking to define how many eye points in the reference system. Use 2 eye points.

Display shows: $+E Y E \quad P T 1$

This will reduce the BOX. Use the 2nd smallest BOX.

By pressing the number keys $(0-9)$ when the " + " is on the display until the desired image sharpness is achieved. Halfstep focus can be achieved by pressing the left upper most key on the keypad (un-marked) as necessary. 
15. Looking at the monitor,

16. Press the ENTER button.

17. Display shows: +EYE PT2

18. Press the ENTER button.

\section{REFERENCE SYSTEM 6}

1. Display shows: ?\#PT 6

2. Press the number 2 key. use the encoders to move the $X-Y$ table so the BOX displayed on the monitor is just inside the left corner of the wafer.

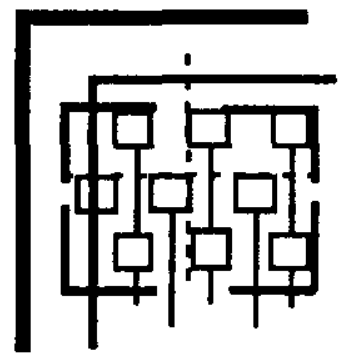

EYE PT 1

Display shows: LEARNING while the $X-Y$ table scans the area in the BOX.

Looking at the monitor, use the encoders to move the $X-Y$ table so the BOX displayed on the monitor is just inside the right corner of the wafer.

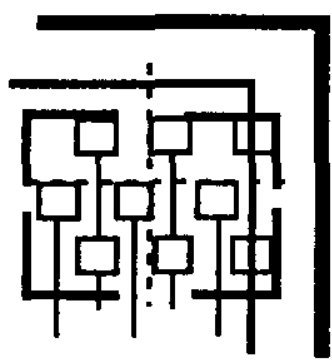

EYE PT 2

Display shows: LEARNING while the $X-Y$ table scans the area in the BOX.

The computer is asking for the number of operator reference points in that will be taught for the sixth reference system. Use 2 reference points.

Display shows: $\quad ? \quad 2=2$ 
3. Press the ACCEPT key.

4. Looking at the monitor,

5. Press the ENTER button.

6. Looking at the monitor,

7. Enter the 2nd operator reference point.

8. Press the ENTER button.

9. Press the ACCEPT key.
Display shows: +REF PTI

The computer is asking for the first operator reference point.

use the encoders to move the $X-Y$ table so the crosshair displayed on the monitor is at the left corner of the trace.

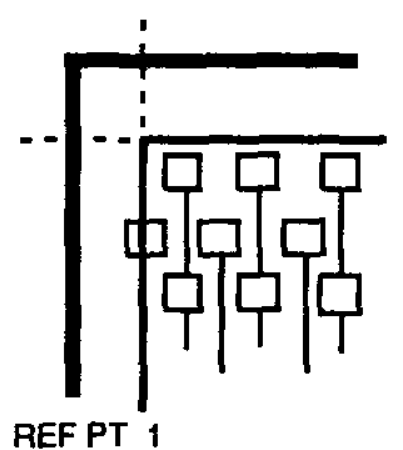

Display shows: +REF PT2 The computer is asking for the second operator reference point.

use the encoders to move the $X-Y$ table so the crosshair displayed on the monitor is at the right corner of the trace.

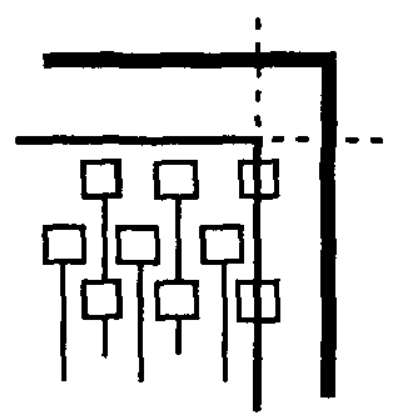

REF PT 2

Display shows: \% DIE TOL The computer is asking for tolerance parameters for the sixth reference system. This has already been defined. 
10. Display shows: ? EYE 2

11. Press the number 2 key.

12. Press the ACCEPT key.

13. Press the Change key.

14. Adjust the focus.

15. Looking at the monitor,

16. Press the ENTER button.
The computer is asking to define how many eye points in the reference system. Use 2 eye points.

Display shows: +EYE PT1

This will reduce the BOX. Use the 2nd smallest BOX.

By pressing the number keys $(0-9)$ when the " + " is on the display until the desired image sharpness is achieved. Halfstep focus can be achieved by pressing the left upper most key on the keypad (un-marked) as necessary.

use the encoders to move the $X-Y$ table so the BOX displayed on the monitor is just inside the left corner of the wafer.

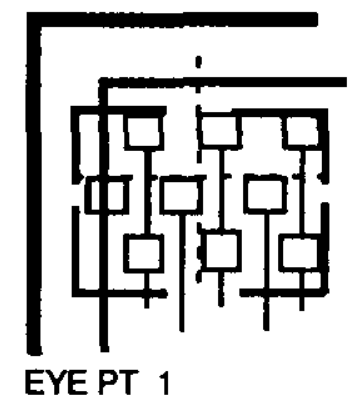

Display shows: LEARNING while the $X-Y$ table scans the area in the BOX. 
17. Display shows: $+E Y E$ PT2

18. Press the ENTER button.

\section{REFERENCE SYSIEM 7}

1. Display shows: ?\#PT 7

2. Press the number 2 key.

3. Press the ACCEPT key.

4. Looking at the monitor,
Looking at the monitor, use the encoders to move the $X-Y$ table so the BOX displayed on the monitor is just inside the right corner of the wafer.

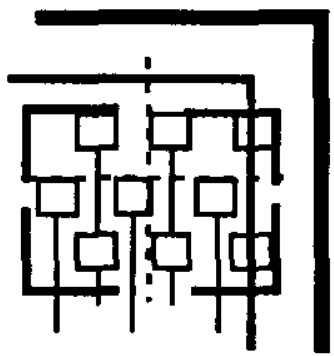

EYE PT 2

Display shows: LEARNING while the $X-Y$ table scans the area in the BOX.

The computer is asking for the number of operator reference points in that will be taught for the seventh reference system. Use 2 reference points.

Display shows: $\quad ? \quad 2=2$

Display shows: $+R E F$ PT1 The computer is asking for the first operator reference point.

use the encoders to move the $X-Y$ table so the crosshair displayed on the monitor is at the left corner of the trace.

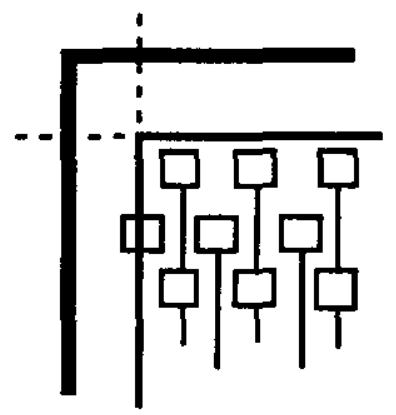

REF PT 1 
5. Press the ENTER button.

6. Looking at the monitor,

7. Enter the 2nd operator reference point.

8. Press the ENTER button.

9. Press the ACCEPT key.

10. Display shows: ? EYE 2

11. Press the number 2 key.

12. Press the ACCEPT key.

13. Press the CHANGE key.

14. Adjust the focus.
Display shows: +REF PT2 The computer is asking for the second operator reference point.

use the encoders to move the $X-Y$ table so the crosshair displayed on the monitor is at the right corner of the trace.

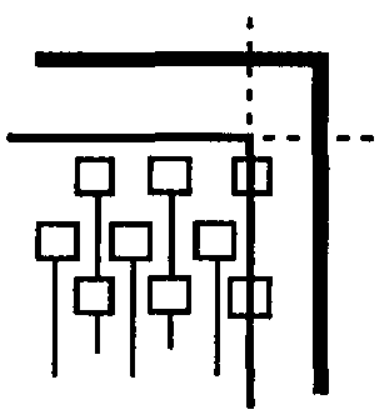

REF PT 2

Display shows: \% DIE TOL The computer is asking for tolerance parameters for the seventh reference system. This has already been defined.

The computer is asking to define how many eye points in the reference system. Use 2 eye points.

Display shows: $+E Y E \quad P T 1$

This will reduce the BOX. Use the 2nd smallest BOX.

By pressing the number keys (0-9) when the " + " is on the display until the desired image sharpness is achieved. Halfstep focus can be achieved by pressing the left upper most key on the keypad (un-marked) as necessary. 
15. Looking at the monitor,

16. Press the ENTER button.

17. Display shows: +EYE PT2

18. Press the ENTER button.

\section{REFERENCE SYSTEM 8}

1. Display shows: ?\#PT 8

2. Press the number 2 key. use the encoders to move the $X-Y$ table so the BOX displayed on the monitor is just inside the left corner of the wafer.

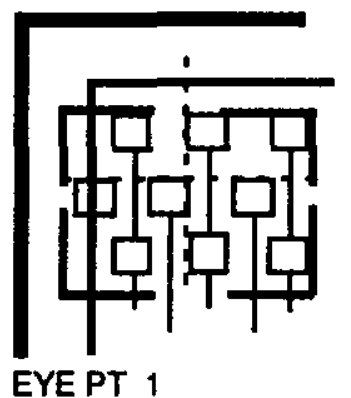

Display shows: LEARNING while the $X-Y$ table scans the area in the BOX.

Looking at the monitor, use the encoders to move the $X-Y$ table so the BOX displayed on the monitor is just inside the right corner of the wafer.

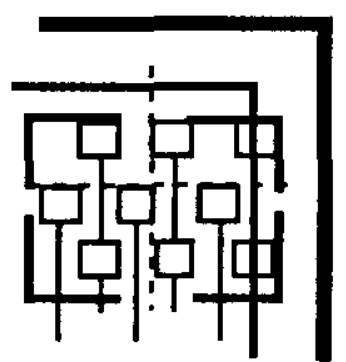

EYE PT 2

Display shows: LEARNING while the $X \cdot Y$ table scans the area in the BOX.

The computer is asking for the number of operator reference points in that will be taught for the eighth reference system.

Use 2 reference points.

Display shows: $\quad ? \quad 2=2$ 
3. Press the ACCEPT key.

4. Looking at the monitor,

5. Press the ENTER button.

6. Looking at the monitor,

7. Enter the 2nd operator reference point.

8. Press the ENTER button.

9. Press the ACCEPT key.
Display shows: +REF PTI The computer is asking for the first operator reference point.

use the encoders to move the $X \cdot Y$ table so the crosshair displayed on the monitor is at the left corner of the trace.

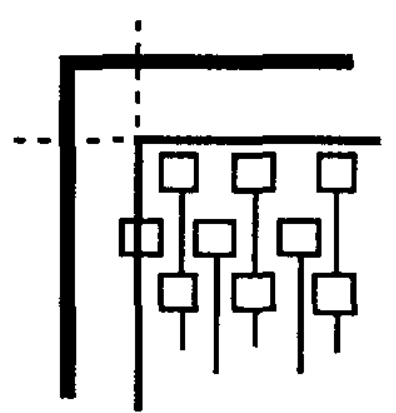

REF PT 1

Display shows: +REF PT2 The computer is asking for the second operator reference point.

use the encoders to move the $X-Y$ table so the crosshair displayed on the monitor is at the right corner of the trace.

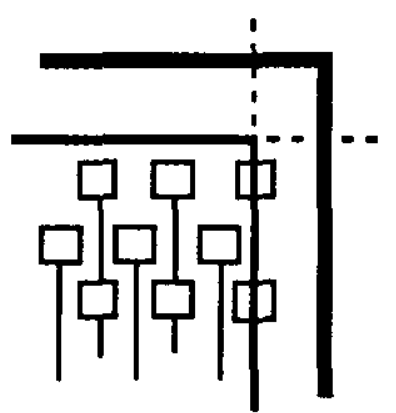

REF PT 2

Display shows: \% DIE TOL The computer is asking for tolerance parameters for the eighth reference system. This has already been defined. 
10. Display shows: ? EYE PT2

11. Press the number 2 key.

12. Press the ACCEPT key.

13. Press the CHANGE key.

14. Adjust the focus.

15. Looking at the monitor.

16. Press the ENTER button.
The computer is asking to define how many eye points in the reference system. Use 2 eye points.

Display shows: +EYE PT1

This will reduce the BOX. Use the 2nd smallest BOX.

By pressing the number keys $(0-9)$ when the " $t$ " is on the display until the desired image sharpness is achieved. Halfstep focus can be achieved by pressing the left upper most key on the keypad (un-marked) as necessary.

use the encoders to move the $X-Y$ table so the BOX displayed on the monitor is just inside the left corner of the wafer.

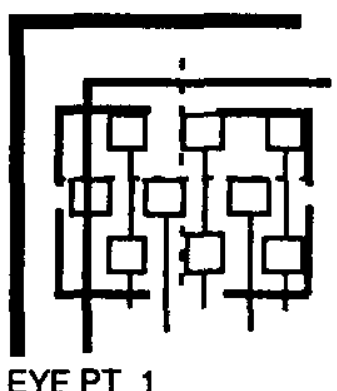

Display shows: LEARNING while the $X-Y$ table scans the area in the BOX. 
Looking at the monitor, use the encoders to move the $X-Y$ table so the BOX displayed on the monitor is just inside the right corner of the water.

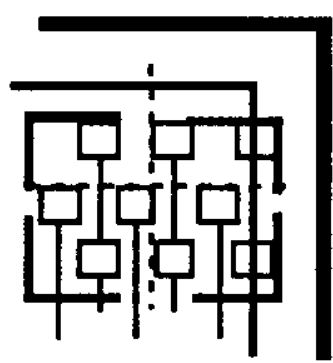

EYE PT 2

18. Press the ENTER button.

Display shows: LEARNING while the $X-Y$ table scans the area in the BOX.

\section{REEERENCE SYSTEM 9}

1. Display shows: ?\#PT 9

2. Press the number 2 key.

3. Press the ACCEPT key,

4. Looking at the monitor,
The computer is asking for the number of operator reference points in that will be taught for the ninth reference system.

Use 2 reference points.

Display shows: ? $2=2$

Display shows: $\quad+R E F \quad P T 1$ The computer is asking for the first operator reference point.

use the encoders to move the $X-Y$ table so the crosshair displayed on the monitor is at the left corner of the trace.

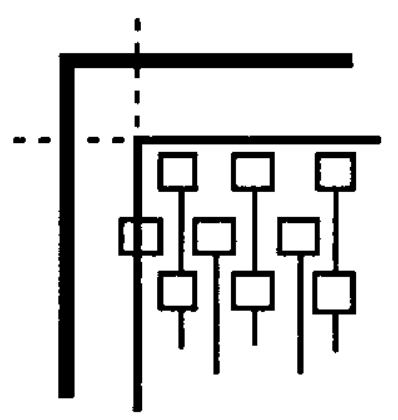

REF PT 1 
5. Press the ENTER button.

6. Looking at the monitor,

7. Enter the 2nd operator reference point.

8. Press the ENTER button.

9. Press the ACCEPT key.

10. Display shows: ? EYE 2

11. Press the number 2 key.

12. Press the ACCEPT key.

13. Press the Change key.
Display shows: +REF PT2 The computer is asking for the second operator reference point.

use the encoders to move the $X \cdot Y$ table so the crosshair displayed on the monitor is at the right corner of the trace.

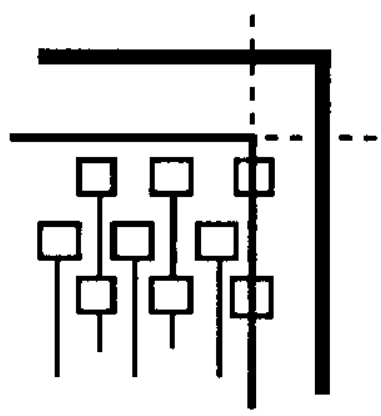

REF PT 2

Display shows: \% DIE TOL The computer is asking for tolerance parameters for the eighth reference system. This has already been defined.

The computer is asking to define how many eye points in the reference system. Use 2 eye points.

Display shows: +EYE PT1

This will reduce the BOX. Use the 2nd smallest BOX. 
14. Adjust the focus.

15. Looking at the monitor,

16. Press the ENTER button.

17. Display shows: +EYE PT2

18. Press the ENTER button.
By pressing the number keys $(0-9)$ when the " + " is on the display until the desired image sharpness is achieved. Halfstep focus can be achieved by pressing the left upper most key on the keypad (un-marked) as necessary.

use the encoders to move the $X-Y$ table so the BOX displayed on the monitor is just inside the left corner of the wafer.

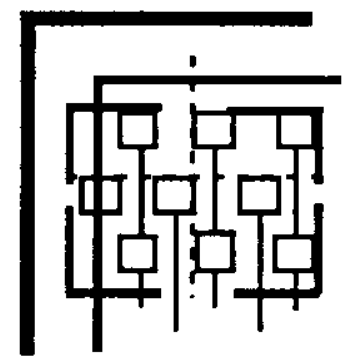

EYE PT 1

Display shows: LEARNING while the $X-Y$ table scans the area in the BOX.

Looking at the monitor, use the encoders to move the $X-Y$ table so the BOX displayed on the monitor is just inside the right corner of the wafer.

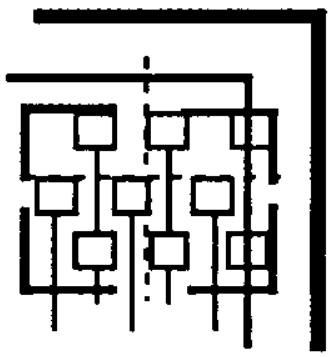

EYE PT 2

Display shows: LEARNING while the $X-Y$ table scans the area in the BOX. 


\section{BEFERENCE SYSTEM 10}

1. Display shows: ?\#PT 10

2. Press the number 2 key.

3. Press the ACCEPT key.

4. Looking at the monitor,

5. Press the ENTER button.

6. Looking at the monitor,
The computer is asking for the number of operator reference points in that will be taught for the tenth reference system. Use 2 reference points.

Display shows: ? $2=2$

Display shows: + REF PT2 The computer is asking for the first operator reference point.

use the encoders to move the $X-Y$ table so the crosshair displayed on the monitor is at the left corner of the trace.

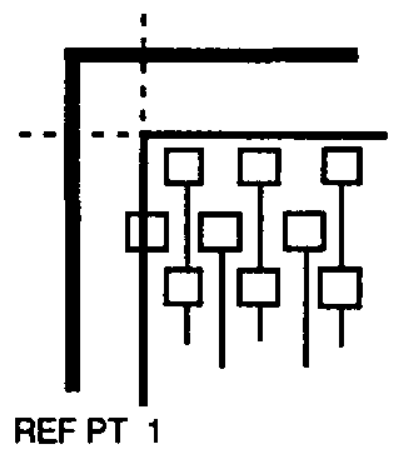

Display shows: +REF PT2 The computer is asking for the second operator reference point.

Use the encoders to move the $X-Y$ table so the crosshair displayed on the monitor is at the right corner of the trace.

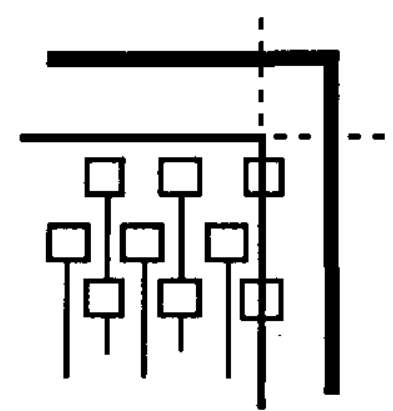

REF PT 2 
7. Enter the 2nd operator reference point.

8. Press the ENTER button.

Display shows: \% DIE TOL The computer is asking for tolerance parameters for the tenth reference system. This has already been defined.

9. Press the ACCEPT key.

10. Display shows: ? EYE 2

The computer is asking to define how many eye points in the reference system. Use 2 eye points.

11. Press the number 2 key.

12. Press the ACCEPT key.

13. Press the CHANGE key.

14. Adjust the focus.

Display shows: + EYE PTI

This will reduce the BOX. Use the 2nd smallest BOX.

By pressing the number keys $(0-9)$ when the " + " is on the display until the desired image sharpness is achieved. Halfstep focus can be achieved by pressing the left upper most key on the keypad (un-marked) as necessary.

15. Looking at the monitor, use the encoders to move the $X \cdot Y$ table so the BOX displayed on the monitor is just inside the left corner of the wafer.

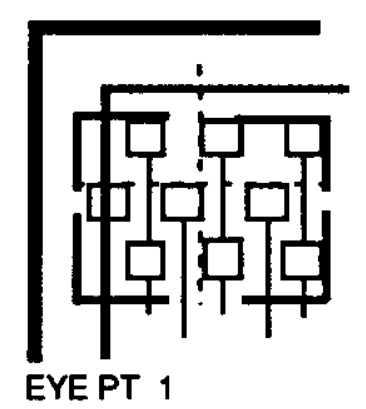

16. Press the ENTER button.

Display shows: LEARNING while the $X-Y$ table scans the area in the BOX. 
17. Display shows: +EYE PT2

18. Press the ENTER button.

\section{REFERENCE SYSTEM 11}

1. Display shows: ?\#PT 11

2. Press the number 2 key.

3. Press the ACCEPT key.

4. Looking at the monitor,
Looking at the monitor, use the encoders to move the X.Y table so the BOX displayed on the monitor is just inside the lower corner of the water.

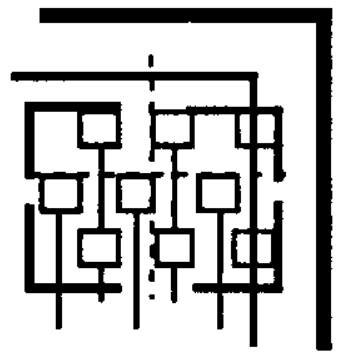

EYEPT 2

Display shows: LEARNING while the $X-Y$ table scans the area in the BOX.

The computer is asking for the number of operator reference points in that will be taught for the eleventh reference system. Use 2 reference points.

Display shows: $\quad 2=2$

Display shows: +REF PT1 The computer is asking for the first operator reference point.

use the encoders to move the $X-Y$ table so the crosshair displayed on the monitor is at the left corner of the trace.

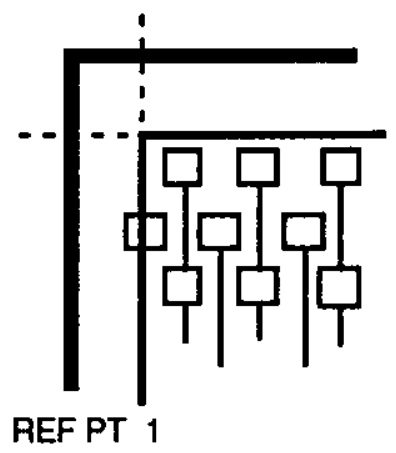


5. Press the ENTER button.

6. Looking at the monitor,

7. Enter the 2nd operator reference point.

8. Press the ENTER button.

9. Press the ACCEPT key.

10. Display shows: ? EYE 2

11. Press the number 2 key.

12. Press the ACCEPT key.

13. Press the CHANGE key.

14. Adjust the focus.
Display shows: + REF PT2 The computer is asking for the second operator reference point.

use the encoders to move the $X-Y$ table so the crosshair displayed on the monitor is at the right corner of the trace.

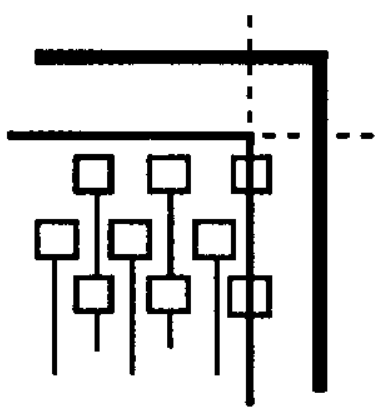

REF PT 2

Display shows: \% DIE TOL The computer is asking for tolerance parameters for the eleventh reference system. This has already been defined.

The computer is asking to define how many eye points in the reference system. Use 2 eye points.

Display shows: $\quad+E Y E \quad P T 1$

This will reduce the BOX. Use the 2nd smallest BOX.

By pressing the number keys $(0-9)$ when the " + " is on the display until the desired image sharpness is achieved. Halfstep focus can be achieved by pressing the left upper most key on the keypad (un-marked) as necessary. 
15. Looking at the monitor,

16. Press the ENTER button.

17. Display shows: $+E Y E \quad P T 2$

18. Press the ENTER button.

\section{REEERENCE SYSTEM 12}

1. Display shows: ?\#PT 12

2. Press the number 2 key. use the encoders to move the $X-Y$ table so the BOX displayed on the monitor is just inside the left corner of the wafer.

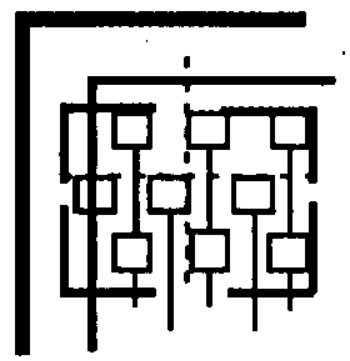

EYE PT 1

Display shows: LEARNING while the $X-Y$ table scans the area in the BOX.

Looking at the monitor, use the encoders to move the X-Y table so the BOX displayed on the monitor is just inside the right corner of the wafer.

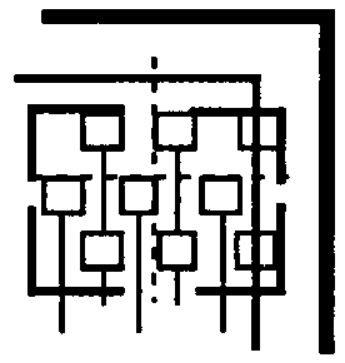

EYE PT 2

Display shows: LEARNING while the $X-Y$ table scans the area in the BOX.

The computer is asking for the number of operator reference points in that will be taught for the twelfth reference system. Use 2 reference points.

Display shows: $\quad ? 2=2$ 
3. Press the ACCEPT key.

4. Looking at the monitor,

5. Press the ENTER button.

6. Looking at the monitor,

7. Enter the 2nd operator reference point.

8. Press the ENTER button.

9. Press the ACCEPT key. display shows: + REF Pr1

The computer is asking for the first operator reference point.

use the encoders to move the $X-Y$ table so the crosshair displayed on the monitor is at the left corner of the trace.

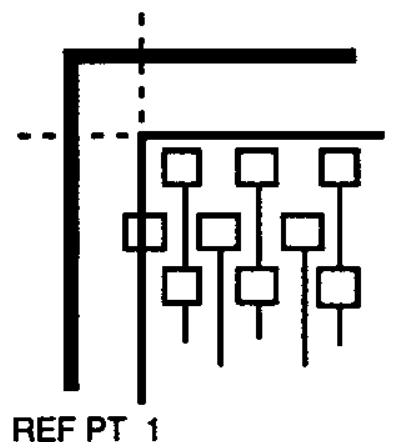

Display shows: +REF PT2 The computer is asking for the second operator reference point.

use the encoders to move the $X-Y$ table so the crosshair displayed on the monitor is at the right corner of the trace.

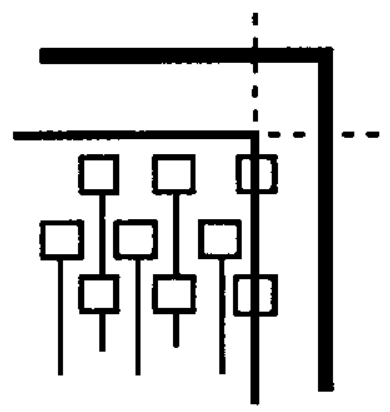

REF PT 2

Display shows: \% DIE TOL The computer is asking for tolerance parameters for the twelfth reference system. This has already been defined. 
10. Display shows: ? EYE 2

11. Press the number 2 key.

12. Press the ACCEPT key.

13. Press the ChANGE key.

14. Adjust the focus.

15. Looking at the monitor,

16. Press the ENTER button.
The computer is asking to define how many eye points in the reference system. Use 2 eye points.

Display shows: + EYE Pr1

This will reduce the BOX. Use the 2nd smallest BOX.

By pressing the number keys $(0-9)$ when the " + " is on the display until the desired image sharpness is achieved. Halfstep focus can be achieved by pressing the left upper most key on the keypad (un-marked) as necessary.

use the encoders to move the $X-Y$ table so the BOX displayed on the monitor is just inside the left corner of the wafer.

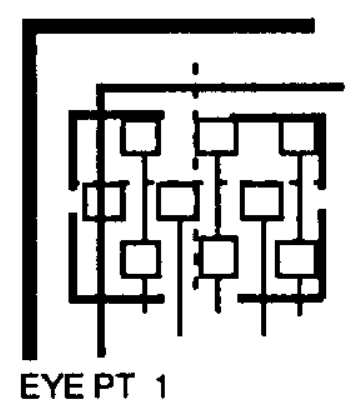

Display shows: LEARNING while the $X-Y$ table scans the area in the BOX. 
17. Display shows: +EYE PT2

18. Press the ENTER button.

\section{REFERENCE SYSTEM 13}

1. Display shows: ?\#PT 13

2. Press the number 2 key.

3. Press the ACCEPT key.

4. Looking at the monitor,
Looking at the monitor, use the encoders to move the $X \cdot Y$ table so the BOX displayed on the monitor is just inside the right corner of the wafer.

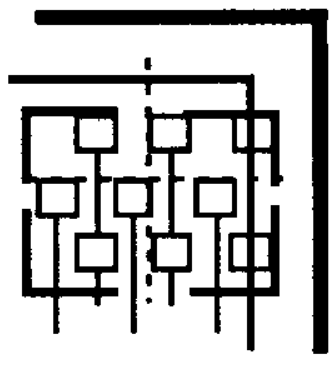

EYE PT 2

Display shows: LEARNING while the $X-Y$ table scans the area in the BOX.

The computer is asking for the number of operator reference points in that will be taught for the thirteenth reference system. Use 2 reference points.

Display shows: ? $2=2$

Display shows: +REF PTI The computer is asking for the first operator reference point.

use the encoders to move the $X-Y$ table so the crosshair displayed on the monitor is at the left corner of the trace.

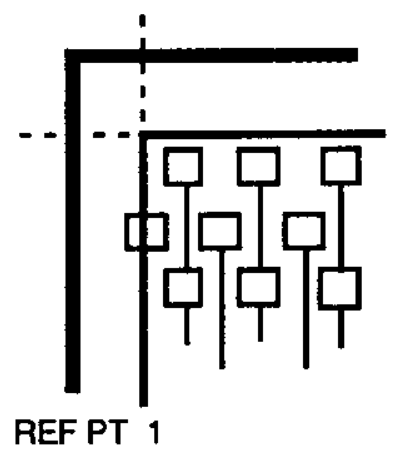


5. Press the ENTER button.

6. Looking at the monitor,

7. Enter the 2nd operator reference point.

8. Press the ENTER button.

9. Press the ACCEPT key.

10. Display shows: ? EYE 2
Display shows: +REF PT2

The computer is asking for the second operator reference point.

use the encoders to move the $X-Y$ table so the crosshair displayed on the monitor is at the right corner of the trace.

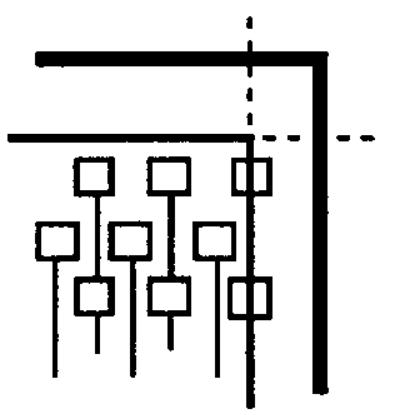

REF PT 2

Display shows: \% DIE TOL The computer is asking for tolerance parameters for the thirteenth reference system. This has already been defined.

The computer is asking to define how many eye points in the reference system. Use 2 eye points.

11. Press the number 2 key.

12. Press the ACCEPT key.

13. Press the CHANGE key.

14. Adjust the focus.
Display shows: +EYE PT1

This will reduce the BOX. Use the 2nd smallest BOX.

By pressing the number keys $(0-9)$ when the " + " is on the display until the desired image sharpness is achieved. Halfstep focus can be achieved by pressing the left upper most key on the keypad (un-marked) as necessary. 
15. Looking at the monitor,

16. Press the ENTER button.

17. Display shows: $+E Y E \quad P T 2$

18. Press the ENTER button.

\section{REEERENCE SYSTEM 14}

1. Display shows: ?\#PT 14

2. Press the number 2 key. use the encoders to move the $X-Y$ table so the BOX displayed on the monitor is just inside the left corner of the wafer.

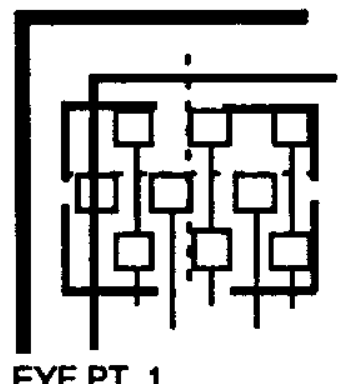

EYE PT 1

Display shows: LEARNING while the $X-Y$ table scans the area in the BOX.

Looking at the monitor, use the encoders to move the $X-Y$ table so the BOX displayed on the monitor is just inside the right corner of the wafer.

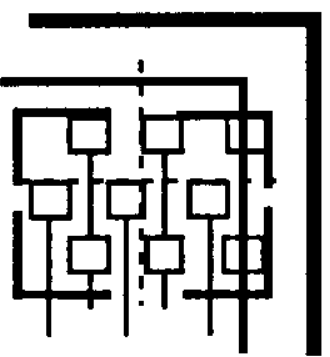

EYE PT 2

Display shows: LEARNING while the $X-Y$ table scans the area in the BOX.

The computer is asking for the number of operator reference points in that will be taught for the fourteenth reference system. Use 2 reference points.

Display shows: ? $2=2$ 
3. Press the ACCEPT key.

4. Looking at the monitor,

5. Press the ENTER button.

6. Looking at the monitor,
Display shows: +REF PT1 The computer is asking for the first operator reference point.

use the encoders to move the $X-Y$ table so the crosshair displayed on the monitor is at the left corner of the trace.

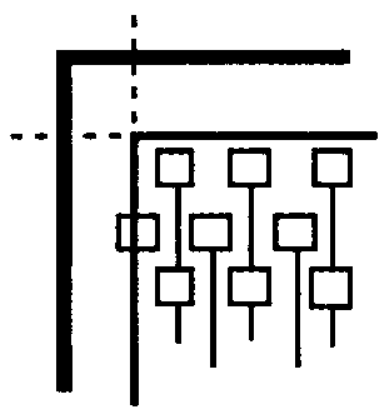

REF PT 1

Display shows: +REF PT2 The computer is asking for the second operator reference point.

use the encoders to move the $X-Y$ table so the crosshair displayed on the monitor is at the right corner of the trace.

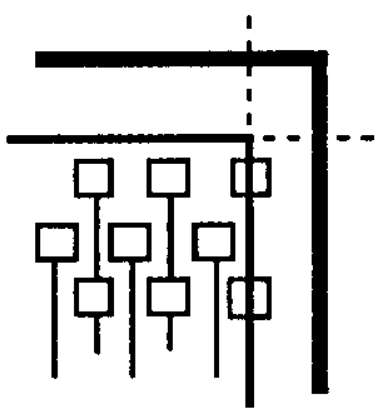

REF PT 2

7. Enter the 2nd operator reference point.

8. Press the ENTER button.

Display shows: \% DIE TOL The computer is asking for tolerance parameters for the twelfth reference system. This has already been defined.

9. Press the ACCEPT key. 
10. Display shows: ? EYE 2

11. Press the number 2 key.

12. Press the ACCEPT key.

13. Press the CHANGE key.

14. Adjust the focus.

15. Looking at the monitor,

16. Press the ENTER button.
The computer is asking to define how many eye points in the reference system. Use 2 eye points.

Display shows: +EYE PT1

This will reduce the BOX. Use the 2nd smallest BOX.

By pressing the number keys $(0-9)$ when the " $t$ " is on the display until the desired image sharpness is achieved. Halfstep focus can be achieved by pressing the left upper most key on the keypad (un-marked) as necessary.

use the encoders to move the $X-Y$ table so the BOX displayed on the monitor is just inside the left corner of the wafer.

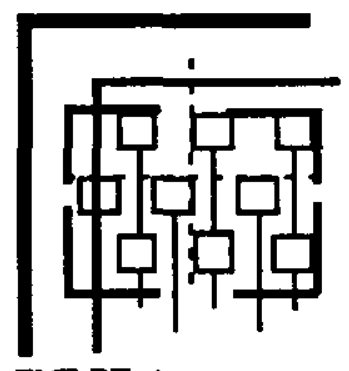

EYE PT 1

Display shows: LEARNING while the $X-Y$ table scans the area in the BOX. 
Looking at the monitor, use the encoders to move the $X-Y$ table so the BOX displayed on the monitor is just inside the right corner of the wafer.

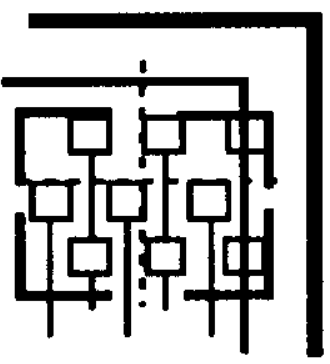

EYE PT 2

18. Press the ENTER button.

Display shows: LEARNING while the $X-Y$ table scans the area in the BOX.

\section{INNER ROWS}

\section{REFERENCE SYSTEMS 1 \& 3}

1. Display shows: ?FRM

Teach the from/to relationship of the wires. Press a number key that represents the reference system where the first bonds will be placed.

2. Press the 1 key,

then press the ACCEPT key. All wires on reference system one are first bonds.

3. Display shows: ?TO

Press the 3 key, then the ACCEPT key. All wires on reference system three are second bonds.

4. Display shows: $+W \quad 1.1$

The computer assumes that the "TO/FROM" relationship of wires and reference systems will be the same until changed. The plus sign means the $X-Y$ table will be moved, the "W" means wire, the first numeral indicates the number of the wire to be bonded, and the second numeral indicates which bond (first(1) or second(2)) will be taught. 
5. Looking at the monitor,

6. To display the loop height of the wires,

7. The current setting for the inner row is \#\#\#.

8. Display shows: \#\#

9. Display shows: IA $1 D$ IW

10. Press the ACCEPT key.

11. Display the contact velocity of the bonding head.

12. Press the 6 key.

13. Press the ACCEPT key.

14. Displays shows: ! $A 101 \mathrm{~W}$

15. Display the loop profile.

16. Display shows: \% CLM TIP

17. Display shows: \% TER

18. Display shows: $+W \quad 1.1$

19. Press the ENTER button. use the encoders to move the $X \cdot Y$ table so the crosshair displayed on the monitor is over the center of the first bond point of wire 1 .

Press the LOOP HEIGHT key. LOOP HEIGHT is different between the inner row and outer row. Consult the LOG. BOOK for current parameters.

Press \# \# keys.

Press the ACCEPT key.

Press the ONE DEVICE key.

Press the SPECIAL FUNCTION key and then the LOOP HEIGHT (CVL) key.

Display shows: 4 . The current setting for CVL is 6 .

Display shows: 6

Press the ONE DEVICE key.

Press the SPECIAL FUNCTION key and then the TIME (CLM) key. The loop profile can be changed by changing the point during the bonding cycle where the wire is clamped. Use the TER position.

Press the CHANGE key until TER is displayed.

Press the ACCEPT key.

Display shows: +w 1.2 
20. Looking at the monitor,

21. Display the contact velocity of the bonding head.

22. Press the 6 key.

23. Press the ACCEPT key.

24. Press the ENTER button.

25. Display shows: $+W 2.1$

26. Looking at the monitor,

27. Press the ENTER button.

28. Looking at the monitor,

29. Press the ENTER button. use the encoders to move the $X-Y$ table so the crosshair displayed on the monitor is over the center of the second bond point of wire 1.

Press the SPECIAL FUNCTION key and then the LOOP HEIGHT (CVL) key.

Display shows: 4 .

This is the contact velocity of the bonding head. The current setting for CVL is 6 .

Display shows: 6

The bonding machine places the first wire. The crosshair returns to bond point 1 for inspection. Use the encoders to move the crosshair to examine bond point 2.

use the encoders to move the $X-Y$ table so the crosshair displayed on the monitor is over the center of the first bond point of wire 2 .

Display shows: +w 2.2

use the encoders to move the $X-Y$ table so the crosshair displayed on the monitor is over the center of the second bond point of wire 2.

The bonding machine places the second wire. The crosshair returns to bond point 1 for inspection. Use the encoders to move the crosshair to examine bond point 2 . 
30. Display shows: $+W 3.1$

\section{REFERENCE SYSTEMS $5 \& 7$}

1. Press the START DEVICE key.

2. Press the 5 key,

3. Display shows: ?TO

4. Display shows: $+W \quad 1.1$

5. Looking at the monitor,

6. To display the loop height of the wires,

7. The current setting for the inner row is \#\#\#.
The display prompts the operator to teach each wire. Follow the prompt until reaching the bottom of the wafer. This is the end of reference systems 1 \& 3 .

Display shows: ?FRM Teach the from/to relationship of the wires. Press a number key that represents the reference system where the first bonds will be placed.

then press the ACCEPT key. All wires on reference system five are first bonds.

Press the 7 key, then the ACCEPT key. All wires on reference system seven are second bonds.

The computer assumes that the "TO/FROM" relationship of wires and reference systems will be the same until changed. The plus sign means the $X-Y$ table will be moved, the " $W$ " means wire, the first numeral indicates the number of the wire to be bonded, and the second numeral indicates which bond (first(1) or second(2)) will be taught.

use the encoders to move the $X-Y$ table so the crosshair displayed on the monitor is over the center of the first bond point of wire 1 .

Press the LOOP HEIGHT key. LOOP HEIGHT is different between the inner row and outer row. Consult the LOG. BOOK for current parameters.

Press \# \# \# keys. 
8. Display shows: \#\#

9. Display shows: !A $10 \mathrm{TW}$

10. Press the ACCEPT key.

11. Display the contact velocity of the bonding head.

12. Press the 6 key.

13. Press the ACCEPT key.

14. Displays shows: ! A 10 IW

15. Display the loop profile.

16. Display shows: \% CLM TIP

17. Display shows: $\%$ TER

18. Display shows: $+W \quad 1.1$

19. Press the ENTER button.

20. Looking at the monitor,

21. Display the contact velocity of the bonding head.

22. Press the 6 key.

23. Press the ACCEPT key.
Press the ACCEPT key.

Press the ONE DEVICE key.

Press the SPECIAL FUNCTION key and then the LOOP HEIGHT (CVL) key.

Display shows: 4 . The current setting for CVL is 6.

Display shows: 6

Press the ONE DEVICE key.

Press the SPECIAL FUNCTION key and then the TIME (CLM) key. The loop profile can be changed by changing the point during the bonding cycle where the wire is clamped. Use the TER position.

Press the CHANGE key until TER is displayed.

Press the ACCEPT key.

Display shows: $+W \quad 1.2$

use the encoders to move the $X-Y$ table so the crosshair displayed on the monitor is over the center of the second bond point of wire 1 .

Press the SPECIAL FUNCTION key and then the LOOP HEIGHT (CVL) key.

Display shows: 4 .

This is the contact velocity of the bonding head. The current setting for CVL is 6 .

Display shows: 6 
24. Press the ENTER button.

25. Display shows: $+W 2.1$

26. Looking at the monitor,

27. Press the ENTER button.

28. Looking at the monitor,

29. Press the ENTER button.

30. Display shows: $+W \quad 3.1$

REFERENCE SYSTEMS $9 \& 11$

1. Press the START DEVICE key.

2. Press the 9 key,
The bonding machine places the first wire. The crosshair returns to bond point 1 for inspection. Use the encoders to move the crosshair to examine bond point 2 .

use the encoders to move the $X-Y$ table so the crosshair displayed on the monitor is over the center of the first bond point of wire 2 .

Display shows: $+w \quad 2.2$

use the encoders to move the $X-Y$ table so the crosshair displayed on the monitor is over the center of the second bond point of wire 2 .

The bonding machine places the second wire. The crosshair returns to bond point 1 for inspection. Use the encoders to move the crosshair to examine bond point 2.

The display prompts the operator to teach each wire. Follow the prompt until reaching the bottom of the wafer. This is the end of reference systems 5 \& 7 .

Display shows: ?FRM teach the from/to relationship of the wires. Press a number key that represents the reference system where the first bonds will be placed.

then press the ACCEPT key. All wires on reference system nine are first bonds. 
3. Display shows: ?TO

4. Display shows: $+W \quad 1.1$

5. Looking at the monitor,

6. To display the loop height of the wires,

7. The current setting for the inner row is \#\#\#.

8. Display shows: \#\#\#

9. Display shows: IA 10 IW

10. Press the ACCEPT key.

11. Display the contact velocity of the bonding head.

12. Press the 6 key.

13. Press the ACCEPT key.

14. Displays shows: IA $1 D$ IW
Press the 1 key twice, then the ACCEPT key. All wires on reference system eleven are second bonds.

The computer assumes that the "TO/FROM" relationship of wires and reference systems will be the same until changed. The plus sign means the X-Y table will be moved, the " $W$ " means wire, the first numeral indicates the number of the wire to be bonded, and the second numeral indicates which bond (first(1) or second(2)) will be taught.

use the encoders to move the $X-Y$ table so the crosshair displayed on the monitor is over the center of the first bond point of wire 1 .

Press the LOOP HEIGHT key. LOOP HEIGHT is different between the inner row and outer row. Consult the LOC. BOOK for current parameters.

Press \# \# \# keys.

Press the ACCEPT key.

Press the ONE DEVICE key.

Press the SPECIAL FUNCTION key and then the LOOP HEIGHT (CVL) key.

Display shows: 4 . The current setting for CVL is 6 .

Display shows: 6

Press the ONE DEVICE key. 
15. Display the loop profile.

16. Display shows: \% CLM TIP

17. Display shows: \% TER

18. Display shows: $+W \quad 1.1$

19. Press the ENTER button.

20. Looking at the monitor,

21. Display the contact velocity of the bonding head.

22. Press the 6 key.

23. Press the ACCEPT key.

24. Press the ENTER button.

25. Display shows: $+W \quad 2.1$

26. Looking at the monitor,

27. Press the ENTER button.
Press the SPECIAL FUNCTION key and then the TIME (CLM) key. The loop profile can be changed by changing the point during the bonding cycle where the wire is clamped. Use the TER position.

Press the CHANGE key until TER is displayed.

Press the ACCEPT key.

Display shows: +w 1.2

use the encoders to move the $X-Y$ table so the crosshair displayed on the monitor is over the center of the second bond point of wire 1 .

Press the SPECIAL FUNCTION key and then the LOOP HEIGHT (CVL) key.

Display shows: 4 .

This is the contact velocity of the bonding head. The current setting for CVL is 6 .

Display shows: 6

The bonding machine places the first wire. The crosshair returns to bond point 1 for inspection. Use the encoders to move the crosshair to examine bond point 2.

use the encoders to move the $X-Y$ table so the crosshair displayed on the monitor is over the center of the first bond point of wire 2 .

Display shows: $+w \quad 2.2$ 
28. Looking at the monitor,

29. Press the ENTER button.

30. Display shows: $+W \quad 3.1$

REFERENCE SYSTEMS $9 \& 13$

1. Press the START DEVICE key.

2. Press the 9 key,

3. Display shows: ?TO

4. Display shows: $+W \quad 1.1$ use the encoders to move the $X-Y$ table so the crosshair displayed on the monitor is over the center of the second bond point of wire 2 .

The bonding machine places the second wire. The crosshair returns to bond point 1 for inspection. Use the encoders to move the crosshair to examine bond point 2 .

The display prompts the operator to teach each wire. Follow the prompt until reaching the bottom of the wafer. This is the end of reference systems 9 \& 11.

Display shows: ?FRM

Teach the from/to relationship of the wires. Press a number key that represents the reference system where the first bonds will be placed.

then press the ACCEPT key. All wires on reference system nine are first bonds.

Press the 13 keys, then the ACCEPT key. All wires on reference system thirteen are second bonds.

The computer assumes that the "TO/FROM" relationship of wires and reference systems will be the same until changed. The plus sign means the $X-Y$ table will be moved, the " $W$ " means wire, the first numeral indicates the number of the wire to be bonded, and the second numeral indicates which bond (first(1) or second(2)) will be taught. 
5. Looking at the monitor,

6. To display the loop height of the wires,

7. The current setting for the inner row is \#\#\#.

8. Display shows: \#\#

9. Display shows: /A $10 \mathrm{IW}$

10. Press the ACCEPT key.

11. Display the contact velocity of the bonding head.

12. Press the 6 key.

13. Press the ACCEPT key.

14. Displays shows: ! A $101 \mathrm{~W}$

15. Display the loop profile.

16. Display shows: \% CLM TIP

17. Display shows: \% TER

18. Display shows: $+W \quad 1.1$

19. Press the ENTER button. use the encoders to move the $X-Y$ table so the crosshair displayed on the monitor is over the center of the first bond point of wire 1 .

Press the LOOP HEIGHT key. LOOP HEIGHT is different between the inner row and outer row. Consult the LOG. BOOK for current parameters.

Press \# \# \# keys.

Press the ACCEPT key.

Press the ONE DEVICE key.

Press the SPECIAL FUNCTION key and then the LOOP HEKGHT (CVL) key.

Display shows: 4 . The current setting for CVL is 6 .

Display shows: 6

Press the ONE DEVICE key.

Press the SPECIAL FUNCTION key and then the TIME (CLM) key. The loop profile can be changed by changing the point during the bonding cycle where the wire is clamped. Use the TER position.

Press the CHANGE key until TER is displayed.

Press the ACCEPT key.

Display shows: +w 1.2 
20. Looking at the monitor,

21. Display the contact velocity of the bonding head.

22. Press the 6 key.

23. Press the ACCEPT key.

24. Press the ENTER button.

25. Display shows: $+W 2.1$

26. Looking at the monitor,

27. Press the ENTER button.

28. Looking at the monitor,

29. Press the ENTER button. use the encoders to move the $X-Y$ table so the crosshair displayed on the monitor is over the center of the second bond point of wire 1 .

Press the SPECIAL FUNCTION key and then the LOOP HEIGHT (CVL) key.

Display shows: 4 .

This is the contact velocity of the bonding head. The current setting for CVL is 6 .

Display shows: 6

The bonding machine places the first wire. The crosshair returns to bond point 1 for inspection. Use the encoders to move the crosshair to examine bond point 2 .

use the encoders to move the $X-Y$ table so the crosshair displayed on the monitor is over the center of the first bond point of wire 2.

Display shows: + w2.2

use the encoders to move the $X-Y$ table so the crosshair displayed on the monitor is over the center of the second bond point of wire 2 .

The bonding machine places the second wire. The crosshair returns to bond point 1 for inspection. Use the encoders to move the crosshair to examine bond point 2 . 
30. Display shows: + W 3.1

\section{OUTER ROWS}

\section{REFERENCE SYSTEMS $2 \& 4$}

1. Press the START DEVICE key.

2. Press the 2 key,

3. Display shows: ?TO

4. Display shows: $+W \quad 1.1$

5. Looking at the monitor,

6. To display the loop height of the wires,
The display prompts the operator to teach each wire. Follow the prompt until reaching the bottom of the wafer. This is the end of reference systems $9 \& 13$.

Display shows: ?FRM Teach the from/to relationship of the wires. Press a number key that represents the reference system where the first bonds will be placed.

then press the ACCEPT key. All wires on reference system two are first bonds.

Press the 4 key, then the ACCEPT key. All wires on reference system four are second bonds.

The computer assumes that the "TO/FROM" relationship of wires and reference systems will be the same until changed. The plus sign means the $X-Y$ table will be moved, the " $W$ " means wire, the first numeral indicates the number of the wire to be bonded, and the second numeral indicates which bond (first(1) or second(2)) will be taught.

use the encoders to move the $X-Y$ table so the crosshair displayed on the monitor is over the center of the first bond point of wire 1 .

Press the LOOP HEIGHT key. LOOP HEIGHT is different between the inner row and outer row. Consult the LOG. BOOK for current parameters. 
7. The current setting for the inner row is \#\#.

8. Display shows: \#\#

9. Display shows: IA $1 D$ IW

10. Press the ACCEPT key.

11. Display the contact velocity of the bonding head.

12. Press the 6 key.

13. Press the ACCEPT key.

14. Displays shows: ! $A$ ID $1 \mathrm{~W}$

15. Display the loop profile.

16. Display shows: \% CLM TIP

17. Display shows: \% TER

18. Display shows: $+W \quad 1.1$

19. Press the ENTER button.

20. Looking at the monitor,

21. Display the contact velocity of the bonding head.

22. Press the 6 key.
Press \# \# keys.

Press the ACCEPT key.

Press the ONE DEVICE key.

Press the SPECIAL FUNCTION key and then the LOOP HEIGHT (CVL) key.

Display shows: 4 . The current setting for CVL is 6 .

Display shows: 6

Press the ONE DEVICE key.

Press the SPECIAL FUNCTION key and then the TIME (CLM) key. The loop profile can be changed by changing the point during the bonding cycle where the wire is clamped. Use the TER position.

Press the CHANGE key until TER is displayed.

Press the ACCEPT key.

Display shows: $+w 1.2$

use the encoders to move the $X-Y$ table so the crosshair displayed on the monitor is over the center of the second bond point of wire 1 .

Press the SPECIAL FUNCTION key and then the LOOP HEIGHT (CVL) key.

Display shows: 4 .

This is the contact velocity of the bonding head. The current setting for CVL is 6 .

Display shows: 6 
23. Press the ACCEPT key.

24. Press the ENTER button.

25. Display shows: $+W \quad 2.1$

26. Looking at the monitor,

27. Press the ENTER button.

28. Looking at the monitor,

29. Press the ENTER button.

30. Display shows: + W 3.1
The bonding machine places the first wire. The crosshair returns to bond point 1 for inspection. Use the encoders to move the crosshair to examine bond point 2 .

use the encoders to move the $X-Y$ table so the crosshair displayed on the monitor is over the center of the first bond point of wire 2 .

Display shows: $+w \quad 2.2$

use the encoders to move the $X-Y$ table so the crosshair displayed on the monitor is over the center of the second bond point of wire 2 .

The bonding machine places the second wire. The crosshair retums to bond point 1 for inspection. Use the encoders to move the crosshair to examine bond point 2 .

The display prompts the operator to teach each wire. Follow the prompt until reaching the bottom of the wafer. This is the end of reference systems $2 \& 4$.

Display shows: ?FRM teach the from/to relationship of the wires. Press a number key that represents the reference system where the first bonds will be placed.

then press the ACCEPT key. All wires on reference system six are first bonds. 
3. Display shows: ?TO

4. Display shows: $+W \quad 1.1$

5. Looking at the monitor,

6. To display the loop height of the wires,

7. The current setting for the inner row is \#\#\#.

8. Display shows: \#\#\#

9. Display shows: / A $1 D$ IW

10. Press the ACCEPT key.

11. Display the contact velocity of the bonding head.

12. Press the 6 key.

13. Press the ACCEPT key.

14. Displays shows: !A $1 D \mathrm{IW}$
Press the 8 key, then the ACCEPT key. All wires on reference system eight are second bonds.

The computer assumes that the "TO/FROM" relationship of wires and reference systems will be the same until changed. The plus sign means the X-Y table will be moved, the " $W$ " means wire, the first numeral indicates the number of the wire to be bonded, and the second numeral indicates which bond (first(1) or second(2)) will be taught.

use the encoders to move the $X-Y$ table so the crosshair displayed on the monitor is over the center of the first bond point of wire 1 .

Press the LOOP HEIGHT key. LOOP HEIGHT is different between the inner row and outer row. Consult the LOG BooK for current parameters.

Press \# \# \# keys.

Press the ACCEPT key.

Press the ONE DEVICE key.

Press the SPECIAL FUNCTION key and then the LOOP HEIGHT (CVL) key.

Display shows: 4 . The current setting for CVL is 6.

Display shows: 6

Press the ONE DEVICE key. 
15. Display the loop profile.

16. Display shows: $\% C L M T I P$

17. Display shows: \% TER

18. Display shows: $+W 1.1$

19. Press the ENTER button.

20. Looking at the monitor,

21. Display the contact velocity of the bonding head.

22. Press the 6 key.

23. Press the ACCEPT key.

24. Press the ENTER button.

25. Display shows: $+W 2.1$

26. Looking at the monitor,

27. Press the ENTER button.
Press the SPECIAL FUNCTION key and then the TIME (CLM) key. The loop profile can be changed by changing the point during the bonding cycle where the wire is clamped. Use the TER position.

Press the CHANGE key until TER is displayed.

Press the ACCEPT key.

Display shows: $+w 1.2$

use the encoders to move the $X-Y$ table so the crosshair displayed on the monitor is over the center of the second bond point of wire 1 .

Press the SPECIAL FUNCTION key and then the LOOP HEIGHT (CVL) key.

Display shows: 4 .

This is the contact velocity of the bonding head. The current setting for CVL is 6 .

Display shows: 6

The bonding machine places the first wire. The crosshair returns to bond point 1 for inspection. Use the encoders to move the crosshair to examine bond point 2.

use the encoders to move the $X-Y$ table so the crosshair displayed on the monitor is over the center of the first bond point of wire 2 .

Display shows: $+W \quad 2.2$ 
28. Looking at the monitor,

29. Press the ENTER button.

30. Display shows: $+W 3.1$

REFERENCE SYSTEMS $10 \& 12$

1. Press the START DEVICE key.

2. Press the 1.0 keys,

3. Display shows: ?TO

4. Display shows: $+W \quad 1.1$ use the encoders to move the $X-Y$ table so the crosshair displayed on the monitor is over the center of the second bond point of wire 2 .

The bonding machine places the second wire. The crosshair retums to bond point 1 for inspection. Use the encoders to move the crosshair to examine bond point 2.

The display prompts the operator to teach each wire. Follow the prompt until reaching the bottom of the wafer. This is the end of reference systems $6 \& 8$.

Display shows: ?FRM teach the from/to relationship of the wires. Press a number key that represents the reference system where the first bonds will be placed.

then press the ACCEPT key. All wires on reference system ten are first bonds.

Press the 12 keys, then the ACCEPT key. All wires on reference system twelve are second bonds.

The computer assumes that the "TO/FROM" relationship of wires and reference systems will be the same until changed. The plus sign means the $X-Y$ table will be moved, the " $W^{*}$ means wire, the first numeral indicates the number of the wire to be bonded, and the second numeral indicates which bond (first(1) or second(2)) will be taught. 
5. Looking at the monitor,

6. To display the loop height of the wires,

7. The current setting for the inner row is \#\#\#.

8. Display shows: \#\#\#

9. Display shows: !A 10 IW

10. Press the ACCEPT key.

11. Display the contact velocity of the bonding head.

12. Press the 6 key.

13. Press the ACCEPT key.

14. Displays shows: ! 10 IW

15. Display the loop profile.

16. Display shows: \% CLM TIP

17. Display shows: \% TER

18. Display shows: $+W \quad 1.1$

19. Press the ENTER button. use the encoders to move the $X-Y$ table so the crosshair displayed on the monitor is over the center of the first bond point of wire 1 .

Press the LOOP HEIGHT key. LOOP HEIGHT is different between the inner row and outer row. Consult the LOC. BOOK for current parameters.

Press \# \# \# keys.

Press the ACCEPT key.

Press the ONE DEVICE key.

Press the SPECIAL FUNCTION key and then the LOOP HEIGHT (CVL) key.

Display shows: 4 . The current setting for CVL is 6 .

Display shows: 6

Press the ONE DEVICE key.

Press the SPECIAL FUNCTION key and then the TIME (CLM) key. The loop profile can be changed by changing the point during the bonding cycle where the wire is clamped. Use the TER position.

Press the CHANGE key until TER is displayed.

Press the ACCEPT key.

Display shows: +w 1.2 
20. Looking at the monitor,

21. Display the contact velocity of the bonding head.

22. Press the 6 key.

23. Press the ACCEPT key.

24. Press the ENTER button.

25. Display shows: $+W 2.1$

26. Looking at the monitor,

27. Press the ENTER button.

28. Looking at the monitor,

29. Press the ENTER button. use the encoders to move the $X-Y$ table so the crosshair displayed on the monitor is over the center of the second bond point of wire 1 .

Press the SPECIAL FUNCTION key and then the LOOP HEIGHT (CVL) key.

Display shows: 4 .

This is the contact velocity of the bonding head. The current setting for CVL is 6 .

Display shows: 6

The bonding machine places the first wire. The crosshair returns to bond point 1 for inspection. Use the encoders to move the crosshair to examine bond point 2 .

use the encoders to move the $X-Y$ table so the crosshair displayed on the monitor is over the center of the first bond point of wire 2 .

Display shows: $+w \quad 2.2$

use the encoders to move the $X-Y$ table so the crosshair displayed on the monitor is over the center of the second band point of wire 2 .

The bonding machine places the second wire. The crosshair returns to bond point 1 for inspection. Use the encoders to move the crosshair to examine bond point 2. 
30. Display shows: $+W 3.1$

\section{REFERENCE SYSTEMS $10 \& 14$}

1. Press the START DEVICE key.

2. Press the 10 keys,

3. Display shows: ?TO

4. Display shows: $+W \quad 1.1$

5. Looking at the monitor,

6. To display the loop height of the wires,

7. The current setting for the inner row is \#\#\#.
The display prompts the operator to teach each wire. Follow the prompt until reaching the bottom of the wafer. This is the end of reference systems $10 \& 12$.

Display shows: ?FRM

Teach the from/to relationship of the wires. Press a number key that represents the reference system where the first bonds will be placed.

then press the ACCEPT key. All wires on reference system ten are first bonds.

Press the 14 keys, then the ACCEPT key. All wires on reference system fourteen are second bonds.

The computer assumes that the "TO/FROM" relationship of wires and reference systems will be the same until changed. The plus sign means the $X-Y$ table will be moved, the "W" means wire, the first numeral indicates the number of the wire to be bonded, and the second numeral indicates which bond (first(1) or second(2)) will be taught.

use the encoders to move the $X-Y$ table so the crosshair displayed on the monitor is over the center of the first bond point of wire 1.

Press the LOOP HEIGHT key. LOOP HEIGHT is different between the inner row and outer row. Consult the LOG BOOK for current parameters.

Press \# \# \# keys. 
8. Display shows: \#\#\#

9. Display shows: !A 10 IW

10. Press the ACCEPT key.

11. Display the contact velocity of the bonding head.

12. Press the 6 key.

13. Press the ACCEPT key.

14. Displays shows: IA 10 IW

15. Display the loop profile.

16. Display shows: $\% C L M T I P$

17. Display shows: \% TER

18. Display shows: $+W \quad 1.1$

19. Press the ENTER button.

20. Looking at the monitor,

21. Display the contact velocity of the bonding head.

22. Press the 6 key.

23. Press the ACCEPT key.
Press the ACCEPT key.

Press the ONE DEVICE key.

Press the SPECIAL FUNCTION key and then the LOOP HEFHT (CVL) key.

Display shows: 4 . The current setting for CVL is 6 .

Display shows: 6

Press the ONE DEVICE key.

Press the SPECIAL FUNCTION key and then the TIME (CLM) key. The loop profile can be changed by changing the point during the bonding cycle where the wire is clamped. Use the TER position.

Press the CHANGE key until TER is displayed.

Press the ACCEPT key.

Display shows: +w 1.2

use the encoders to move the $X-Y$ table so the crosshair displayed on the monitor is over the center of the second bond point of wire 1 .

Press the SPECIAL FUNCTION key and then the LOOP HEIGHT (CVL) key.

Display shows: 4 .

This is the contact velocity of the bonding head. The current setting for CVL is 6 .

Display shows: 6 
24. Press the ENTER button.

25. Display shows: $+W 2.1$

26. Looking at the monitor,

27. Press the ENTER button.

28. Looking at the monitor,

29. Press the ENTER button.

30. Display shows: $+W \quad 3.1$

31. Press the END key when all wires are placed.

32. Press a mode key or,
The bonding machine places the first wire. The crosshair returns to bond point 1 for inspection. Use the encoders to move the crosshair to examine bond point 2 .

use the encoders to move the $X . Y$ table so the crosshair displayed on the monitor is over the center of the first bond point of wire 2 .

Display shows: $+w \quad 2.2$

use the encoders to move the $X-Y$ table so the crosshair displayed on the monitor is over the center of the second bond point of wire 2 .

The bonding machine places the second wire. The crosshair returns to bond point 1 for inspection. Use the encoders to move the crosshair to examine bond point 2.

The display prompts the operator to teach each wire. Follow the prompt until reaching the bottom of the wafer. This is the end of reference systems $10 \& 14$.

Display flashes: the computer ID and TAUGHT.

Press the EMERGENCY STOP button. The button will shine and the $\mathrm{Z}$ rod solenoid can be heard latching. 


\section{FLOPPY MODE}

1. To save the program,

Press the EMERGENCY STOP button.

2. Place an uniormatted disk in the disk drive and rotate the load/unload lever down.

3. Press the FLOPPY key.

Display shows: ! PLY/REC

4. Press the RECORD key.

Display shows: BUSY

The computer is checking that the disk driver has power.

5. Display shows: ?PRGM 10

Use numeral keys to identify the program.

6. Press the ACCEPT key.

Display shows: $R E C O R D^{\prime} G$ the program is being recorded onto the disk.

7. The display alternately flashes PROGRAM RECORDED

When the program has been copied onto the floppy disk. The program is also still in the computer until a new program is taught or the bonder is powered down.

8. Press a mode key or,

Press the EMERGENCY STOP button. The button will shine and the $Z$ rod solenoid can be heard latching. 